\title{
Correction to: Tuning dual three-dimensional porous copper/graphite composite to achieve diversified utilization of copper current collector for lithium storage
}

Xia Ma, Zhi-Jia Zhang*(i), Jia-Min Wang, Shi-Hao Sun, Shao-Fei Zhang, Shen Yuan, Zhi-Jun Qiao, Zhen-Yang Yu, Jian-Li Kang, Wei-Jie Li

(C) Youke Publishing Co., Ltd. 2021

\section{Correction:}

Rare Met.

https://doi.org/10.1007/s12598-020-01684-y

In the original publication, incomplete Fig. 1 has been published. The correct version of Fig. 1 is provided in this The original article has been corrected. correction.

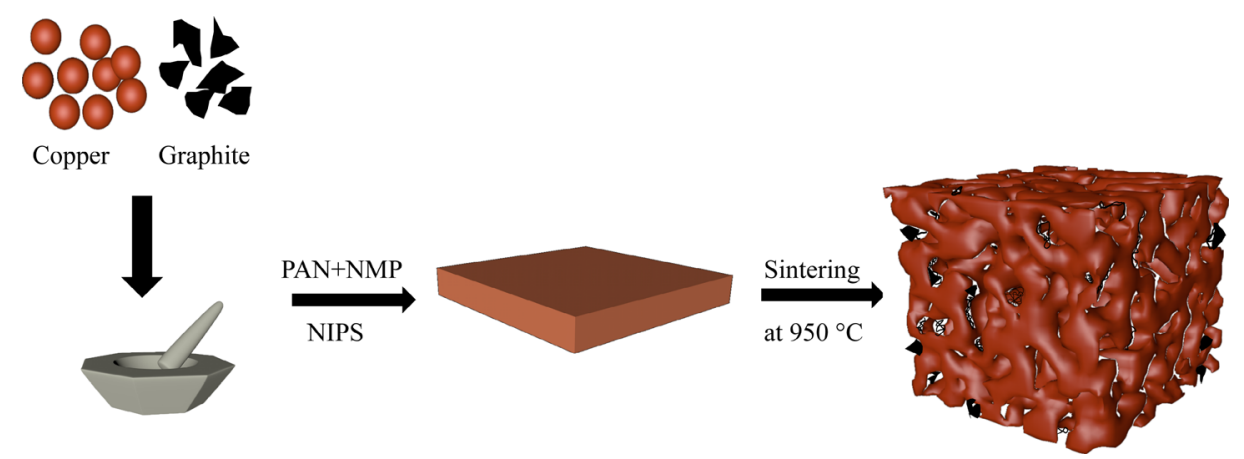

Fig. 1 Schematic illustration for preparation process of 3DP-Cu/graphite film

The original article can be found online at https://doi.org/10.1007/ s12598-020-01684-y.

X. Ma, Z.-J. Zhang*, J.-M. Wang, S.-H. Sun, S.-F. Zhang,

S. Yuan, Z.-J. Qiao, Z.-Y. Yu, J.-L. Kang

State Key Laboratory of Separation Membrane and Membrane

Processes, Tianjin Municipal Key Laboratory of Advanced

Fibers and Energy Storage, School of Materials Science and

Engineering, School of Mechanical Engineering, Tiangong

University, Tianjin 300387, China

e-mail: zhangzhijia@tiangong.edu.cn

\section{Ma}

School of Materials Science and Engineering, Tianjin

University, Tianjin 300072, China
S.-F. Zhang

School of Materials Science and Engineering, Hebei Key Laboratory of Material Near-Net Forming Technology, Hebei University of Science \& Technology, Shijiazhuang 050018, China

W.-J. Li

Institute for Superconducting and Electronic Materials, University of Wollongong, Wollongong 2522, Australia 